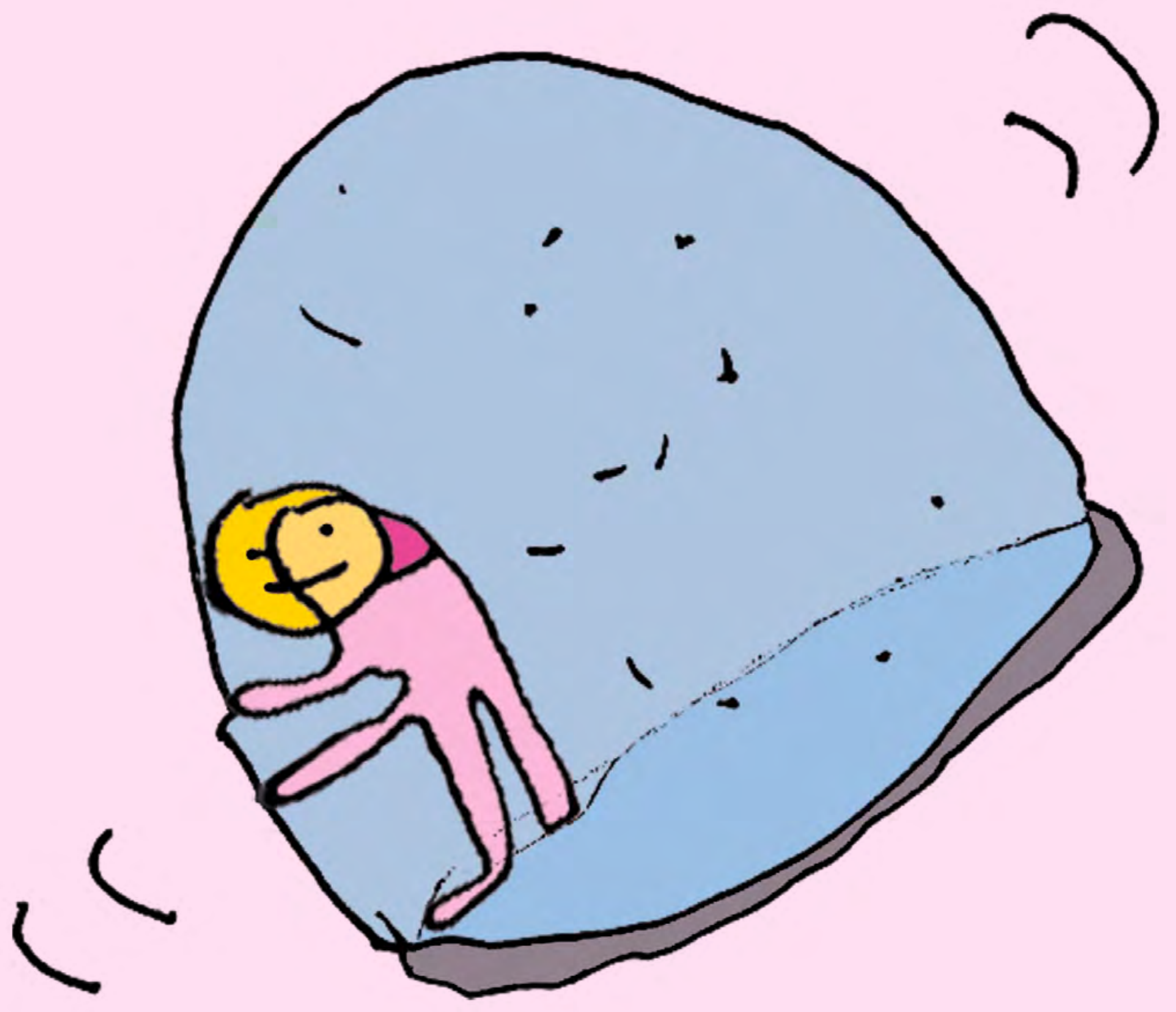


Una mirada

a las revistas

especializadas

en el campo de la

industria creativa

Érika Castañeda Rivera

Bianca Garduño Bello 


\section{Una mirada a las revistas especializadas en el campo de la industria creativa}

\section{Érika Castañeda Rivera}

castaneda.erika@outlook.com | Universidad Iberoamericana, México.

\section{Bianca Garduño Bello}

bigarduno@gmail.com | El Colegio de México, México.

Fecha de recepción: 27 de noviembre de 2017 | Fecha de aceptación: 16 de abril de 2018

\section{Resumen}

En los últimos años, las industrias creativas han mostrado un crecimiento y una participa-ción cada vez más importante en la economía mundial, lo cual es un fenómeno que tiene implicaciones políticas, económicas y sociales que exigen ser reflexionadas, estudiadas y analizadas desde diferentes perspectivas. Para la investigación en ciencias sociales, el tema está abierto y está siendo tratado con especial interés. En este sentido, las revistas especia-lizadas son un espacio fundamental de difusión de la información y el conocimiento que se está generando sobre el campo. En este texto se propone explorar las revistas y publicacio-nes periódicas de la investigación en ciencias sociales para conocer los temas y debates que se están trabajando.

Palabras clave | industrias creativas, revistas especializadas, difusión, ciencias sociales, investigación. 


\begin{abstract}
In recent years, the creative industries have shown growth and participation becoming increasingly crucial in the global economy, which is a phenomenon that has political, economic and social implications that demand to be reflected, studied and analyzed from different perspectives. For research in the social sciences, the subject is open and treated with particular interest. In this sense, journals are a central space to disseminate the information and knowledge generated. This text aims to explore the magazines and periodicals of social science research to learn about the issues and debates that are working.
\end{abstract}

Keywords | specialized journals, creative industries, broadcasting, social sciences, research. 


\section{Introducción}

En el mundo, las industrias creativas han adquirido mayor atención recientemente pues el trabajo que se está realizando en las profesiones que le conforman está implicando mayor participación en la economía mundial y, como consecuencia, muchos cambios en las dinámicas sociales y políticas de su entorno. Estas industrias generan empleos e impulsan la actividad profesional de quienes ofrecen sus productos o servicios; en 2013, la industria empleó a 29 millones de personas y obtuvo ganancias por más de 2 mil millones de dólares, según los datos del reporte de EY referente al mapeo mundial de industrias culturales y creativas (2015). En México, las industrias creativas tienen importantes implicaciones en la economía: el 7\% del PIB, de acuerdo con datos del Instituto Mexicano para la Competitividad (IMCO, 2015), provino de sectores relacionados con la producción de bienes y servicios creativos, una cifra que seguramente irá en aumento y sobre la que es preciso reflexionar tanto acerca de las ventajas (generación de empleo, diversificación de la producción, ampliación de campo laboral para nuevas profesiones), como de los efectos que pueden suponer riesgos (precarización de las condiciones laborales, generación de mercados informales, entre otros).

Una de las razones por las que el crecimiento y la expansión de la industria ha sido tan acelerado en las últimas décadas es que ha sido impactada fuertemente por los efectos de la tecnología y las nuevas formas de comunicación. La generación de conocimiento y la mejora en la técnica son la fuente del avance en las economías nacionales porque el flujo de información permite que el intercambio, tanto local como internacional, genere competencia e innovación para el desarrollo (Porter, 2007). En este ámbito es donde el internet y los medios digitales han funcionado como una de las principales plataformas de difusión de contenidos de los productos que son resultado del trabajo creativo, lo que permite que sean alcanzables y estén disponibles con la inmediatez que caracteriza nuestro tiempo.

Las ciencias sociales no han sido ajenas a los efectos de la industria, como tampoco han sido ajenas a las innovaciones de la tecnología en los medios de comunicación. En este sentido, los temas de investigación relacionados con la industria creativa son relativamente nuevos, 
pero el interés por conocerla, estudiarla y generar datos e información se ha extendido se ha extendido como tema de investigación y el medio fundamental para la difusión de los trabajos, resultados, discusiones y debates son las revistas electrónicas de investigación académica. Para las ciencias sociales, las revistas científicas son el medio principal donde se difunde el trabajo (Cano, 1995; Patalano, 2005) y justamente las dinámicas de publicación han sido uno de los ámbitos donde las nuevas formas de comunicación, la tecnología y el internet han tenido efectos transformadores. Es el uso del espacio virtual el que ha revolucionado la forma de publicar resultados de investigación, compartir información y abrir las posibilidades de conocer a la industria creativa, medirla, caracterizarla, regularla y proponer las guías para su desarrollo.

Las revistas electrónicas como espacio de divulgación es de lo más reciente en la producción de conocimiento sobre la industria y ha sido objeto de estudio desde algunas perspectivas y con objetivos específicos. Muchos de los trabajos que se han realizado sobre las revistas electrónicas, de carácter bibliométrico, ofrecen datos para el análisis de la producción científica que sirven como indicadores de la capacidad nacional de producir información (Cano, 1995). Ya sean estudios comparados o localizados en la producción de un país, se trata de mediciones que presentan balances, avances y pendientes de la actividad editorial.

En América Latina, estos estudios son importantes en la medida que ofrecen un panorama crítico de la situación actual de las publicaciones y de la investigación. En el tema de las industrias creativas, dado que es un tema relativamente novedoso para las ciencias sociales, resulta útil hacer una exploración que ubique los acercamientos que se están haciendo, quiénes están planteando los temas, es decir, qué disciplinas y perspectivas, qué están discutiendo, con qué objetivos y cuáles son las agendas actuales.

En el presente artículo, se propone realizar una exploración de las revistas de investigación en ciencias sociales donde se están tratando temas de la industria creativa en América Latina. Consideramos importante acotar la mirada en América Latina dado que es un contexto donde la investigación académica es aún marginal (Ochoa, 2004), sin embargo, 
existen espacios y esfuerzos significativos para impulsar su desarrollo. El presente se aleja de ser un estudio de corte bibliométrico, y se acerca a una mirada exploratoria de cuatro de las principales hemerotecas virtuales de habla hispana - Latindex (Sistema Regional de Información en Línea para Revistas Científicas de América Latina, el Caribe, España y Portugal), Dialnet (base de datos de revistas científicas en castellano), Redalyc (Red de Revistas Científicas de América Latina y el Caribe, España y Portugal) y ARLA (Asociación de Revistas Latinoamericanas de Arquitectura) - en busca de las revistas en ciencias sociales donde se da espacio a la investigación vinculada con el conocimiento de las industrias creativas y su producción, de tal forma que sea posible identificar los espacios virtuales, los temas y las perspectivas que las ponen como centro de su interés.

Este documento se estructura en tres apartados donde se tratan, primero, los datos de los buscadores donde se hizo la exploración y, a grandes rasgos, cuál es su función y cómo organizan la información de las revistas; segundo, las perspectivas y los debates que se están tratando en la investigación académica en ciencias sobre las industrias creativas y que se observaron con mayor recurrencia en las revistas exploradas; y tercero, una reflexión sobre la importancia de las revistas académicas como espacios de difusión para las industrias creativas así como de la presencia de las mismas como objeto de investigación en las ciencias sociales.

\section{Las industrias creativas en las revistas académicas}

La labor académica que se realiza en los centros de investigación de las universidades, principalmente, tiene como funciones sustantivas la docencia, la generación de conocimiento mediante la investigación científica y su difusión. En América Latina, las últimas décadas se han caracterizado porque las políticas en educación superior han enfatizado la evaluación de procesos y resultados y la instauración de esquemas de financiamiento complementario basados en la productividad (Rodríguez, 2002). En este contexto, la investigación se ha convertido en un indicador del trabajo de los académicos, que en la búsqueda de obtener la transferencia de estímulos económicos condicionados por la medición y evaluación de su 
productividad, concentran gran parte de su esfuerzo en la publicación de sus trabajos en algún medio avalado para la obtención de dichas retribuciones que funcionan de manera compensatoria ante la asignación de salarios bajos a la docencia.

Bajo estas condiciones, los académicos e investigadores han encontrado en las revistas académicas un espacio no sólo para hacer público el producto de su trabajo sino para acumular evidencia, que se traduce en puntaje, para hacerse acreedores de los estímulos económicos o transferencias condicionadas. Con este propósito, las revistas académicas funcionan como plataforma para la divulgación del conocimiento, están sujetas a una serie de requerimientos para cumplir con estándares de calidad y ser consideradas como espacios evaluables. Los criterios que tienen que cumplir para ser consideradas científicas, de calidad y tener estatus de fiabilidad, además de ser un medio útil para los académicos, forman parte de un estándar que comparten índices de revistas como Redalyc, Dialnet, Arla y Latindex, en el contexto de habla hispana, para ser incluidas en sus contenidos. Estos servidores de sistemas de información fueron creados con el objetivo de funcionar como hemerotecas digitales que ponen a disposición pública —Open Access - los productos de la investigación académica. A diferencia de otros servidores que ofrecen contenidos por medio de suscripciones, estos buscadores son de acceso público y gratuito. Además, cuentan con un catálogo muy general, que incluye una vasta diversidad revistas especializadas en todas las disciplinas del conocimiento y tanto aquellas que están arbitradas -es decir, su contenido es evaluado por expertos tanto internos como externos a la institución que las publica- como las que no lo están.

Con el propósito de explorar las revistas de investigación en ciencias sociales que tratan temas vinculados con el desarrollo de la industria creativa, se hizo una búsqueda en los cuatro sistemas de información mencionados y se organizó en cuatro sectores que comprenden las siguientes profesiones: arquitectura, artes escénicas: música, teatro, danza; comunicación y medios: tradicionales y digitales (cine, televisión, radio, prensa, internet); artes visuales: pintura, fotografía, diseño gráfico, diseño industrial, diseño digital, diseño textil. 
En estos cuatro sectores se encontraron más de 36,000 revistas listadas, aunque no representan el total de las revistas, pues hay que anotar que, con excepción de ARLA que se especializa en arquitectura, los otros tres índices incluyen muchas revistas compartidas. Con esta revisión se pudo observar que existe una cantidad importante de publicaciones dedicadas a estudiar los diferentes sectores de la industria. El conjunto de revistas que se revisó (87 revistas de los 4 sectores mencionados y de varios países de habla hispana en América) son primordialmente electrónicas - aunque algunas de ellas cuentan con tirajes impresos-, son arbitradas y están activas, es decir, los números más recientes son de 2016 y 2017.

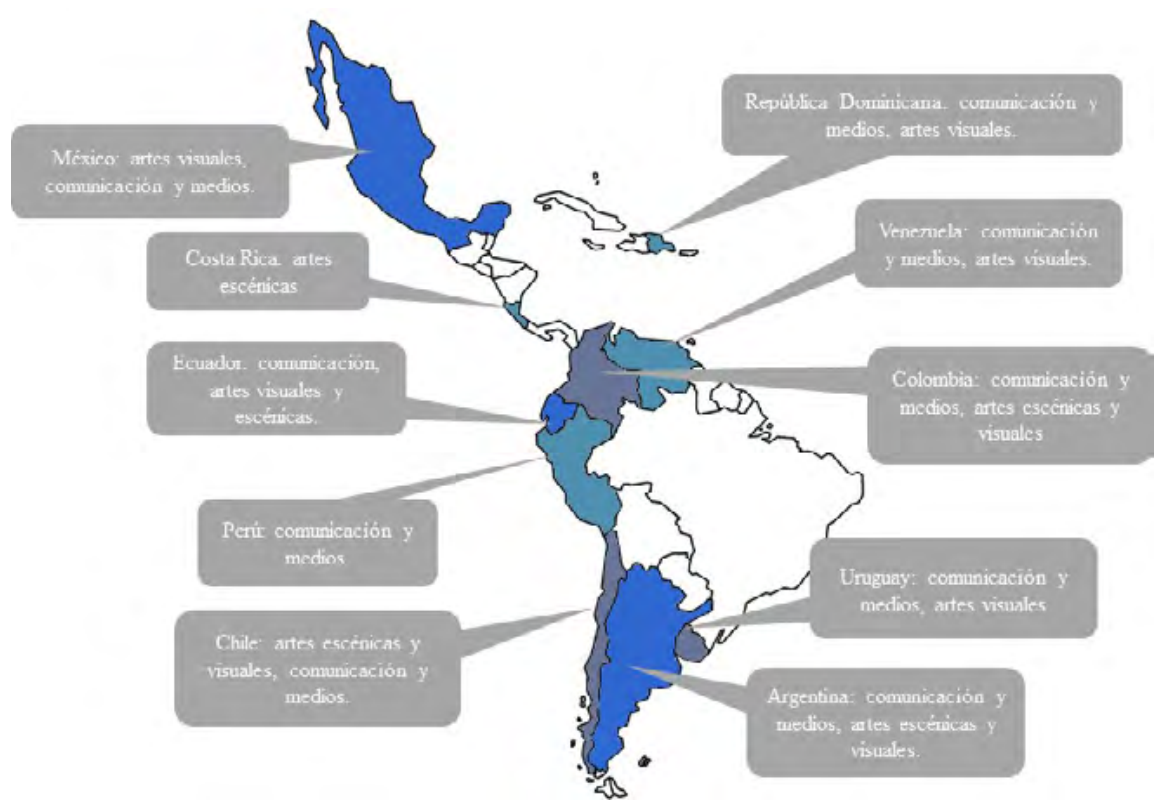

Figura 1. Mapa de revistas en América Latina. (Fuente: Elaboración propia).

Los índices consultados mostraron una producción amplia de revistas que está en constante cambio entre aquellas vigentes y las que suspenden su publicación. Latindex es el índice más extenso cuyo directorio rebasa las 25 mil revistas, le sigue Dialnet con más de 10 mil revistas, Redalyc con más de mil y ARLA con 78 revistas. 


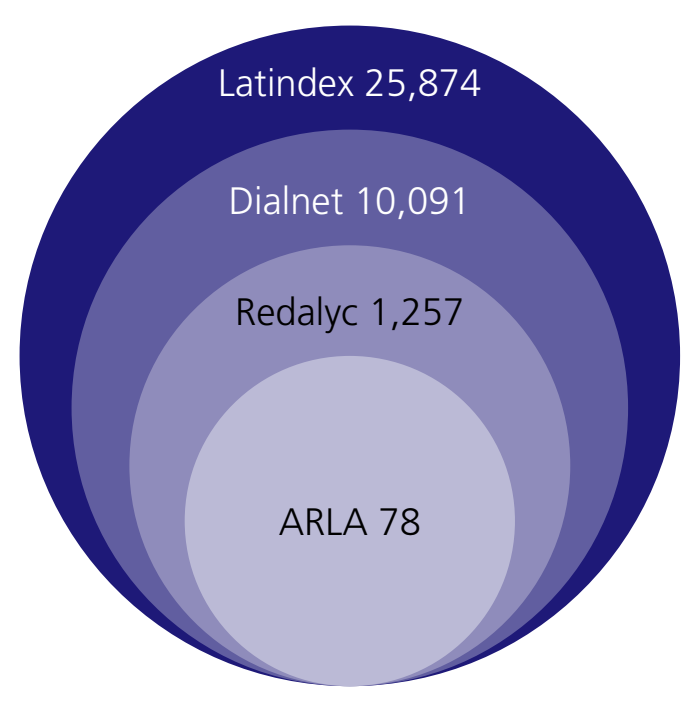

Figura 2. Total de revistas por buscador consultado. (Fuente: Elaboración propia).

Esta revisión mostró que Argentina, Chile, Colombia y México tienen una producción amplia de revistas especializadas y, aunque no es el universo total, es un primer acercamiento a la extensión total de las revistas en América Latina. Cabe señalar que estos países se encuentran entre los primeros 50 con mayor influencia cultural de acuerdo con US News (2017), en un listado de 80 países del mundo. Otros países que están produciendo revistas especializadas son Uruguay, Ecuador, Perú, Costa Rica, República Dominicana y Venezuela.

En esta revisión, se observó que el sector del diseño — gráfico, textil e industrial— es uno de los más difundidos sobre todo en México, ya que, de acuerdo con ProMéxico (2015) el diseño mexicano se encuentra en uno de sus mejores momentos, pues diversos profesionistas del sector han logrado trascender internacionalmente. También, el sector de la comunicación y medios ha demostrado su influencia que se ve reflejada en las publicaciones revisadas: en México existen más de 1,500 productoras entre servicios digitales y de animación, donde está involucrada también a la industria del diseño; además, es el sexto país que exporta más contenidos de animación, videojuegos, software y contenido digital (ProMéxico, 2015). 
La arquitectura y las artes visuales también son sectores con mucha difusión en estas revistas y, de acuerdo con lo revisado, Argentina publica más contenidos relacionados con las artes visuales y a las artes escénicas.

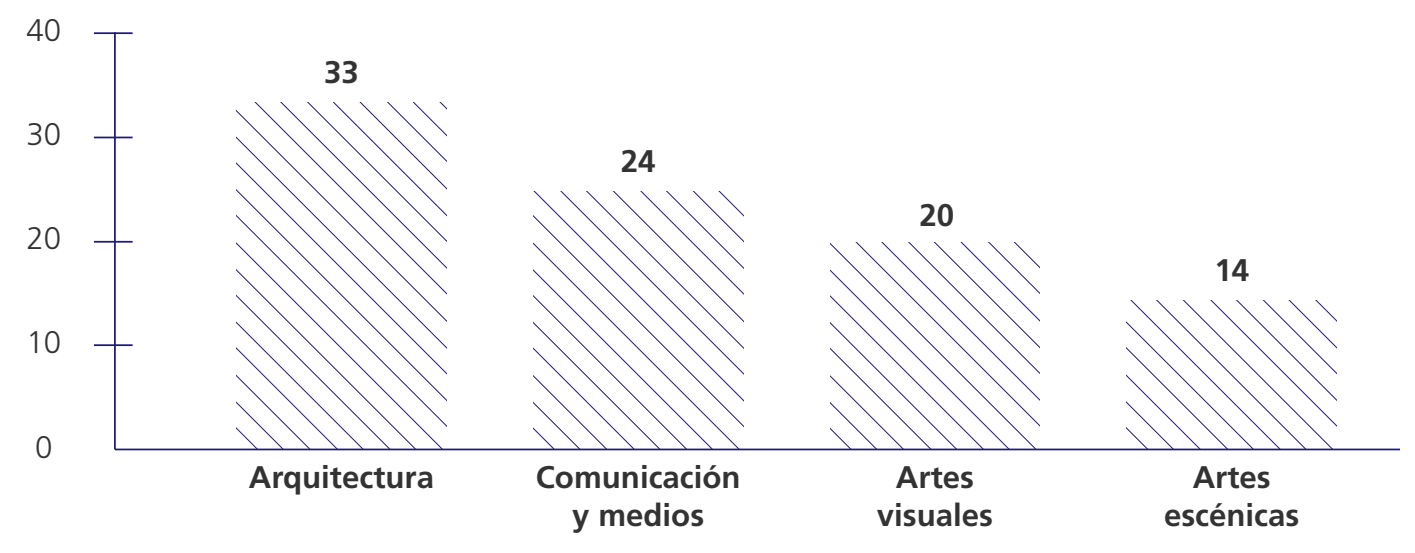

Gráfica 1. Número de revistas por sector de la industria creativa. (Fuente: Elaboración propia con información de Latindex, Dialnet y ARLA).

Es importante mencionar que la mayor parte de las publicaciones revisadas incluyen varios sectores de la industria creativa; es posible encontrar una revista donde se da espacio a los contenidos de artes visuales, artes escénicas, arquitectura o a la comunicación y medios.

\section{Principales temas en debate y perspectivas en México y Latinoamérica}

El acercamiento a las revistas especializadas permitió tener un panorama general de distintas temáticas concretamente en América Latina. De acuerdo con los criterios de búsqueda y los sectores considerados de la industria algunas revistas están enfocadas a realizar publicaciones sobre investigaciones en el campo de la industria creativa en donde se discuten avances y nuevas perspectivas de análisis sobre determinados temas. También se encontró que no solo se difunde la investigación sino que además hay propuestas para aplicar los conocimientos hallados en el campo de la industria y también están aquellas que se apoyan de otras dimensiones para realizar análisis, investigación o plantear algunas líneas de reflexión, algunos contenidos realizan sus aportaciones desde perspectivas políticas, 
económicas y sociales con lo cual contribuyen a la generación de conocimiento dentro de las industrias creativas y ayudan a comprender su impacto en la sociedad actual.

A continuación se hace una revisión general de los temas de debate en América Latina, es importante señalar que la revisión de temas no sugiere que la revista se especializa únicamente en los temas vinculados con las industrias creativas. Por el contrario, la gran mayoría de las revistas nos se limita a dichos temas, sino a una diversidad más amplia en el espectro de las ciencias sociales y humanidades. Sin embargo, lo que aquí se presenta, se limita a la exposición de las que se han abierto a la divulgación de contenidos de la industria creativa.

\section{1) Arquitectura}

Un tema recurrente para este sector es el que tiene que ver con la ecología y la sustentabilidad, se percibe un interés en el cuidado del ambiente y es un tema relativamente nuevo que está generando nuevos conocimientos a partir de la investigación puesto que se considera un tema de impacto mundial. Aquí las revistas de los países de América Latina revisados para este artículo coinciden en la relevancia del tema y algunas revistas realizan el análisis desde el tema de la planeación urbana, la utilización del territorio a favor de los espacios verdes y la sustentabilidad, un término muy recurrente en los artículos de distintas revistas de este sector. AUS, revista chilena, aborda temas como arquitectura sostenible, bioclimática y humanitaria, mientras que Estudios del Hábitat de Argentina y Acta Universitaria de México plantean el estudio de este sector desde la transformación urbana, los cambios sociales y geopolíticos. Por otro lado, Gremium de México estudia la conservación del patrimonio a partir de la sustentabilidad, urbanismo, sociología urbana, ingenierías y ciencia de materiales.

Otro tema considerado dentro del análisis de las revistas de arquitectura se centra en los derechos humanos, lo cual refleja la situación mundial actual y que se relaciona con temas de etnicidad, migración e inclusión social. En algunas revistas mexicanas se observa la preocupación sobre la construcción del muro fronterizo así que la reflexión se relaciona con las adecuaciones en torno a la planeación urbana, particularmente relacionados con el derecho de vivienda y espacios seguros para migrantes. La Revista Invi de Chile analiza temas 
de movilidad social, desplazamiento, políticas públicas y exclusión e inclusión social como justificación de la edificación. Bitácora, revista mexicana en su número Fronteras, dedica sus páginas al debate de la migración, al análisis de la construcción de muro fronterizo y la identidad nacional desde la arquitectura. Bitácora urbano-territorial de Colombia, también aborda el tema de la exclusión social desde la perspectiva de la construcción y ordenamiento urbano. También existen distintas revistas que dedican sus páginas a la arquitectura desde los nuevos procesos de construcción hasta los materiales y diseños más novedosos actualmente.

\section{2) Artes escénicas}

En esta revisión se encontraron diversas revistas que se vinculan a los sectores de la música, el teatro y la danza. En muchas de ellas se menciona la importancia de la cultura diversa y estas publicaciones han abierto espacios para la proyección de la identidad y la representación de la multiculturalidad a través de las artes. Algunas publicaciones que difunden estos temas es Cuadernos de música, artes visuales y artes escénicas de Colombia que discute precisamente sobre la cultura colectiva, El oído pensante de Argentina, que incluso analiza a la música desde la antropología y Epistemus, revista también argentina que estudia la música desde la sociología, antropología, comunicación, historia y psicología.

Una anotación importante es que las revistas dedicadas a las artes escénicas incluyen contenidos dedicados a las artes visuales como el caso de Tsantsa de Ecuador que realiza estudios y análisis en ambos tipos de arte.

\section{3) Comunicación y medios}

El campo de la comunicación y los medios tanto tradicionales como digitales es muy diverso. Por ejemplo, en México las revistas que se especializan en comunicación dedican espacios para reflexionar sobre la crisis de libertad de expresión y los derechos humanos, ya que actualmente el país es uno de los más peligrosos para ejercer el periodismo ${ }^{1}$ o para tener la oportunidad de pensamiento y expresión divergentes; como ejemplo de este tipo

1 Con información del NY Times disponible en https://www.nytimes.com/es/2017/04/29/matar-periodistas-mexico-veracruz/ 
de publicaciones están las páginas de Global Media Journal y la Revista del Centro de Investigación. En Venezuela se refleja una evidente preocupación por la democratización de los medios como se ha difundido en Quorum académico.

Otros temas relevantes en este sector son la comunicación organizacional y la investigación de la comunicación interna y externa. Acerca de estos, una revista que ha abierto un espacio importante para la discusión es Austral Comunicación de Argentina.

También se encuentran los temas de estudios de género: igualdad y violencia, derechos, estereotipos, etc. En México se estudian particularmente estos temas debido al momento que vive el país. En un estudio de la Fundación Thomson Reuters sobre las ciudades más peligrosas para las mujeres, la Ciudad de México se encuentra dentro de las diez primeras y resalta la situación de la violencia sexual². La preocupación por los derechos de las mujeres es un tema vigente y México es la prueba de que la problemática es grave y requiere atención inmediata, tanto de la sociedad civil, como de las autoridades para proponer soluciones desde el nivel legislativo. Algunas de las revistas dedicadas a este tipo de temas son Comunicación y sociedad de México y en Ecuador está la revista Chasqui.

Algunos contenidos se apoyan en otras ciencias para realizar investigaciones y aportaciones más completas, tal es el caso de Cuadernos de $\mathrm{H}$ ideas de Argentina que incluye aproximaciones desde disciplinas como la antropología, el derecho o la sociología.

\section{4) Artes visuales}

En este sector y de acuerdo con la revisión realizada para este artículo, se encuentran publicaciones multidisciplinarias que combinan los temas de artes visuales con comunicación, arquitectura o artes escénicas. Es interesante observar que muchas de ellas incluyen alguna de estas combinaciones, lo cual hace que las revistas sean ricas en información de diferentes disciplinas. Los sectores del diseño -desde el industrial, textil hasta el digital- la

2 Estudio completo disponible en http://poll2017.trust.org/ 
pintura y la fotografía quedan expuestos desde diferentes perspectivas a través de otras artes y disciplinas. En este caso, las revistas de las artes visuales resultaron ser aquellas de contenido más variado. Por ejemplo, la revista Cuadernos del Centro de Estudios en Diseño y Comunicación de Argentina, difunde contenido del sector de las artes visuales combinado con un análisis desde los medios de comunicación; El Ornitorrinco Tachado de México, integra estudios sobre temas de diseño desde la sociología, la familia, la colonia y las organizaciones sociales; y RChD: creación y pensamiento de Chile combina el diseño industrial con el gráfico. Algunas revistas que dedican sus páginas al diseño textil, lo hacen desde la antropología o lo vinculan con otras artes como Iconofacto de Colombia que realiza estudios desde la historia y los significados sociales de la vestimenta. También en las artes visuales se trata el tema del trabajo independiente y sus diferentes implicaciones; un ejemplo de algunos artículos a considerar están en Taller Servicio 24 Horas de México.

Además de estos grandes temas hay otros de interés interno como las legislaciones, políticas del mercado de la industria creativa, contenidos específicos en medios e información relevante de las profesiones creativas. Cada uno de los números se convierte en una referencia de información que puede ser aplicada en otros contextos y replicada para nuevos acercamientos.

\section{La economía creativa y su relación con el acercamiento a las revistas especializadas de la industria}

A manera de cierre de esta exploración, cabe hacer un par de comentarios sobre los hallazgos encontrados en las revistas de investigación académica acerca de las industrias creativas. Por un lado, hay que destacar la importancia de que las revistas académicas abran un espacio para la visibilidad de las industrias creativas que incluyen un espectro amplio de profesiones donde están presentes procesos de conocimiento, de enseñanza y aprendizaje, de formación de talento, de capacitación para la innovación, y de la aplicación de la creatividad en acciones concretas. En este sentido, los espacios virtuales para la publicación de información resultan fundamentales por la inmediatez del intercambio de conocimiento que puede generar y promover el desarrollo de la industria creativa. Así pues, las revistas funcionan 
como un medio de exposición y difusión de sus productos y avances mediante la aplicación del conocimiento y el medio virtual resulta fundamental por la fluidez de sus dinámicas.

Por otro lado, las revistas académicas en internet son fundamentales como medio de difusión de los productos de investigación de las ciencias sociales, y de las dinámicas de retribución de la productividad en el ámbito de la investigación académica. En este tema, las revistas académicas tienen un papel de suma importancia para la divulgación de los productos de investigación bajo criterios de calidad internacional. Es decir, se convierten en los espacios de difusión de conocimiento confiable sobre la industria creativa. Los datos y la información verificable que sea útil para analizar, replicar y proponer soluciones a los efectos y que implique el desarrollo de la industria es lo que se requiere para orientar el desarrollo de la industria hacia la mejor calidad de la producción y también hacia las mejores condiciones de quienes producen.

El número de revistas que se publican en línea ha crecido con el paso del tiempo. A lo largo de esta revisión es posible observar que algunas revistas se originaron en versiones impresas, varios años atrás, cuando no existían los medios digitales. Es decir, el mercado de distribución y de consumo está cambiando, de modo que la industria ha tenido que adaptarse a las nuevas dinámicas editoriales.

En nuestros días, gracias al crecimiento de internet, existe una oferta de contenidos más plural y cada vez más independientes de las grandes compañías editoriales que, durante mucho tiempo, fueron conquistando más espacios traspasando fronteras (Boix y López, 2006). En ellos se configuran distintos discursos y debates en torno a la industria creativa con una peculiaridad, que la digitalización los ha convertido en expresiones culturales fácilmente transportables y manipulables (Durán y Sánchez, 2008).

Estas nuevas estrategias configuran un mercado de información que tiene como común denominador una nueva economía, como lo afirman Boix y López (2006), que no está asociada a la monetaria, sino a la de los seguidores y visitantes. El objetivo central sería acercar a la población y, concretamente, a estudiantes y egresados de carreras de la industria 
creativa a considerar que estos espacios son parte de su formación profesional, que están a su alcance y que existe la posibilidad de contribuir a la acumulación de conocimiento.

Finalmente, este acercamiento a las revistas especializadas brinda un panorama básico del estado de la industria creativa en Latinoamérica en donde es posible observar que existen diversas fuentes de análisis, reflexión y construcción del conocimiento. Además, ofrece una mirada a las distintas aproximaciones a los temas específicos que interesan a cada país. 


\section{Referencias}

Ahmed, Azam (29 de abril, 2017). Es muy fácil matar periodistas: La crisis de la libertad de expresión en México. The New York Times. https://www. nytimes.com/es/2017/04/29/matar-periodistasmexico-veracruz/

ARLA (S/F) Asociación de Revistas Latinoamericanas de Arquitectura. Recuperado de http://arlared. org/?page_id=2

Boix, A. y López, G. (2006). La autoría en la era digital: industria cultural y medios de comunicación. Valencia, España: Tirant lo Blanch.

Cano, V. (1995). Publishing infrastructure of peripheral countries: A comparison of Latin American, USA and UK periodical publications. Scientometrics. $34(1), 121-138$.

Castañeda, E. y Garduño, B. (2016). Mapa de las industrias creativas en México: proyección para Centro. Economía Creativa, 7, 117-166.

Dialnet (S/F) Sistema abierto de información de revistas publicadas. Recuperado de https://dialnet.unirioja.es/

Duran, J. y Sánchez, L. (2008). Industrias de la comunicación audiovisual. Barcelona, España: Edicions de la Universitat de Barcelona.

EY (2015). The First Global Map of Cultural and Creative Industries. Recuperado de http://www. worldcreative.org/

Instituto Mexicano para la Competitividad (2015). Industrias creativas y obra protegida. México: IMCO.

Latindex (S/F) Sistema regional de Información en línea para revistas científicas de América Latina, El Caribe, España y Portugal. Recuperado de http://www.latindex.org/latindex/inicio

Marina, J. A. y Satrústegui, S. (2013). La creatividad económica. Barcelona, España: Ariel.
Naciones Unidas (2010). Economía creativa: Una opción factible de desarrollo. PNUD, UNCTAD.

Ochoa Henríquez, Haydée. (2004) Visibilidad: El reto de las Revistas Científicas Latinoamericanas. Opción, 20 (43), 162-168.

Ordorika Sacristán, I., Lozano Espinosa, F., Rodríguez Gómez, R. (2009). Las revistas de investigación de la UNAM: Un panorama general. Cuadernos de Trabajo de la Dirección General de Evaluación Institucional, No. 4.

Panzano, J. V., y Caño, I. (1998). Revistas electrónicas en ciencias sociales y humanidades. Revista española de documentación científica, 21(3).

Patalano, M. (2005). Las publicaciones del campo científico: las revistas académicas de América Latina.

Porter, M. (2007). La ventaja competitiva de las naciones. Harvard Business Review. América Latina, pp:3-23.

ProMéxico (2015). Mexican design adding values across sectors. Negocios, VII.

Redacción (19 de octubre, 2017). CDMX es la sexta megaciudad más peligrosa para las mujeres en el mundo. El Financiero. Recuperado de http://www. elfinanciero.com. $\mathrm{mx} / \mathrm{mundo} / \mathrm{cdmx}$-es-la-sextamegaciudad-mas-peligrosa-para-las-mujeres-enel-mundo-reuters.html

Rodríguez, R. (2002). Continuidad y cambio en las políticas de educación superior, Revista Mexicana de Investigación Educativa, 7, 14, 133-154.

Román, A. R., Valero, M. V., y Caminos, C. U. (2002). Los criterios de calidad editorial Latindex en el marco de la evaluación de las revistas españolas de humanidades y ciencias sociales. Revista española de documentación científica, 25(3), 286-307. 
Tavares de Matos Cardoso, M. M. (2011). El peer review de las revistas científicas en Humanidades y Ciencias Sociales: políticas y prácticas editoriales declaradas. Revista española de Documentación Científica, 34(2), 141-164.

https://doi.org/10.3989/redc.2011.2.796
Thomson Reuters Foundation (2017). The world's most dangerous megacities for women. Recuperado de http://poll2017.trust.org/

US News. (2017). Best countries. Cultural influence ranks. Recuperado de https://www.usnews.com/ news/best-countries/influence-full-list 